\title{
Fever Responses Are Enhanced with Advancing Age during Respiratory Syncytial Virus Infection among Children under 24 Months Old
}

\author{
Chiaki Kawakami, ${ }^{1}$ Atsuo Sato, ${ }^{2}$ Hiroko Sumita, ${ }^{3}$ Atsushi Isozaki, ${ }^{4}$ \\ Hiroyuki Shimizu, ${ }^{5}$ Taichi Kanetaka, ${ }^{6}$ Koji Maehara, ${ }^{7}$ Kota Ao, ${ }^{8}$ Akiyoshi Nariai, ${ }^{9}$ \\ Fumihiko Takeshita, ${ }^{10}$ Rika Kizu${ }^{1}$ and Masaaki Mori ${ }^{11}$
}

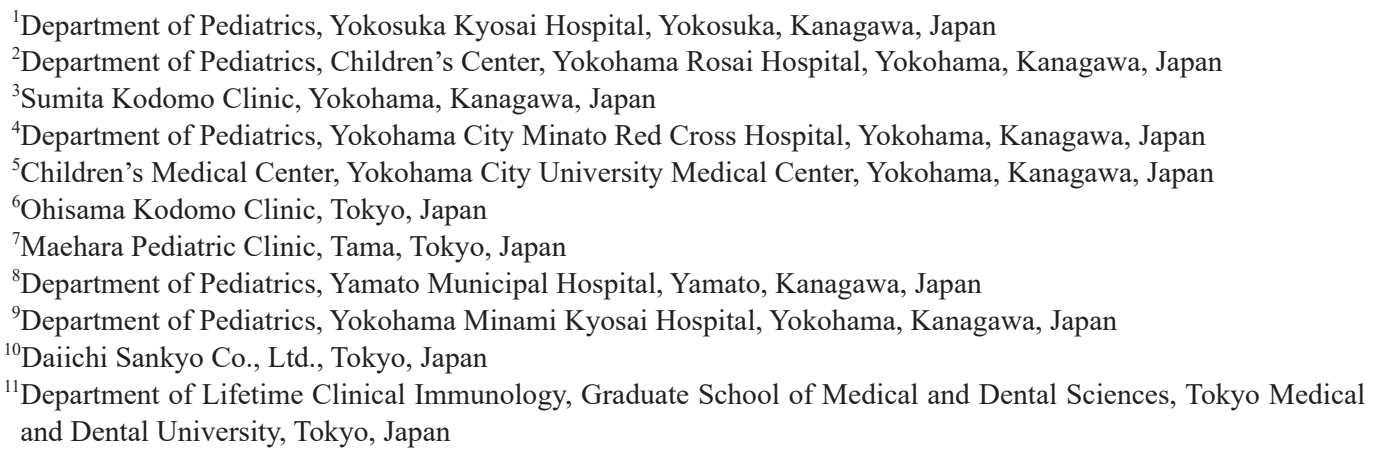

The most important risk factor for severe respiratory syncytial virus (RSV) infection is considered young age due to the immature immune system. The risk at young age is reported greater for RSV than for other respiratory infectious agents. Based on the strong association between young age and severity of RSV infection due to immature immunity, we aimed to assess whether there were any age-related differences in fever responses, as one clinical aspect of the immune response. In our observational study over two seasons (2014-2015 and 2015-2016), daily body temperatures of children under 3 years old with RSV infection were recorded from the first medical visit during the acute phase to defervescence. The body temperature records were analyzed among 171 children of four age groups $(<6,<12,<24$ and $\geq 24$ months), in terms of fever development, degrees of fever onset, the highest fever during the period, and fever duration. There were 54 patients in the group of $<6$ months, 41 in the group of $<12$ months, 58 in the group of $<24$ months, and 18 in the group of $\geq 24$ months. We thus found the correlation between age and fever responses under 24 months old; namely, the more the age advanced, the more frequently high and prolonged fever was experienced. Importantly, infants under 6 months old tend to show the suppressed fever responses. In conclusion, young infants with reduced fever response during RSV infection do not implicate less severity and needs attentive management.

Keywords: fever; fever duration; fever responses; pediatrics; respiratory syncytial virus

Tohoku J. Exp. Med., 2018 July, 245 (3), 217-222. (C) 2018 Tohoku University Medical Press

\section{Introduction}

Almost all children will have experienced a respiratory syncytial virus (RSV) infection by the time they reach 2 years of age (Crowe 2016). Specific underlying diseases are recognized as aggravating factors contributing to sever- ity. However, most patients who suffer from severe consequences of RSV infection are previously healthy children (Hall et al. 2009, 2013; El Saleeby et al. 2011). The most important risk factor related to severity is considered young age (Hall et al. 2009, 2013), compared with other respiratory infectious diseases (El Saleeby et al. 2011). It has been

Received February 13, 2018; revised and accepted July 5, 2018. Published online July 24, 2018; doi: 10.1620/tjem.245.217.

Correspondence: Chiaki Kawakami, Department of Pediatrics, Yokosuka Kyosai Hospital, 1-16 Yonegahama-dori, Yokosuka, Kanagawa 238-8558, Japan.

*Present address: Field Epidemiology Training Program (FETP), Infectious Disease Surveillance Center, National Institute of Infectious

Diseases, 1-23-1 Toyama, Shinjuku-ku, Tokyo 162-8640, Japan.

e-mail: ckawakami25@gmail.com

Masaaki Mori, M.D. Ph.D., Department of Lifetime Clinical Immunology, Graduate School, Tokyo Medical and Dental University, 1-545 Yushima, Bunkyo-ku, Tokyo 113-8510, Japan.

e-mail: mori.phv@tmd.ac.jp 
proposed that such a feature results from the immaturity of infant immune systems (Mella et al. 2013). Several reports have described differences in the production or relative proportions of cytokines as factors related to severity (Welliver 2000; Legg et al. 2003; Amanatidou et al. 2009).

In the clinical setting, although there are few practical parameters that can be taken to reflect immune responses, fever presentation can be considered one such. Based on the proposal that the especially strong association of young age and severity of disease is related to the immaturity of the immune system in RSV infections, we investigated whether there were any age-related differences in fever responses.

\section{Methods}

\section{Study enrollment}

We conducted a prospective observational study of children $<3$ years of age with RSV infections who visited 9 participating hospitals during the two seasons 2014-2015 and 2015-2016. Children with suspicion of RSV infection were assessed using RSV rapid diagnostic tests (Quick Navi ${ }^{\mathrm{TM}}$-RSV; DENKA SEIKEN Co., Ltd. Tokyo, Japan. BD RSV Examan ${ }^{\mathrm{TM}}$; Nippon Becton Dickinson Co., Ltd. Tokyo, Japan. Quick Chaser ${ }^{\circledR}$ RSV; MIZUHO MEDY Co., Ltd. Saga, Japan. ImmunoAce $^{\circledR}$ RSV Neo; TAUNS, Shizuoka, Japan. PRIMECHECK ${ }^{\circledR}$ RSV; Alfresa Pharma Co., Osaka, Japan). Those with confirmed RSV infection according to these diagnostic tests were recruited to this study following their parents' agreement. Exclusions were: a) previous administration of palivizumab, b) a diagnosis of hepatitis B, hepatitis $\mathrm{C}$, syphilis, or HIV infection, c) participation in another clinical trial, or d) considered to be inappropriate for this study by the clinicians.

\section{Patient characteristics and clinical symptoms}

At an enrollment during the acute phase, we asked participants' parents to complete an interview form about patients' age, sex, clinical symptoms at that time, and any specific medication use within the preceding three months, such as immunosuppressants, radiation therapy, anti-rheumatoid drugs, hematinic drugs, corticosteroids, gammaglobulins, or any kind of vaccine.

Clinical symptoms and specific medication use were again asked at the visit during the convalescent phase, which was defined as between two and three weeks after the first acute phase visit.

\section{Fever responses}

Fever was defined as body temperature $\geq 37.5^{\circ} \mathrm{C}$. Defervescence was defined as body temperature remaining $<37.5^{\circ} \mathrm{C}$ for at least 24 hours.

The timing and degree of fever onset were reported at the enrollment. Since the enrollment, parents had recorded axillary body temperature three times a day for fourteen days or until defervescence. The time for measuring body temperature was set at around each mealtime.

Based on the fever onset information and body temperature records, we collected data about whether a patient had developed fever, degree of fever onset, the highest body temperature during the period, and fever duration.
Statistical analysis

Patients were stratified into four age groups: $<6$ months, $\geq 6$ $<12$ months, $\geq 12<24$ months, and $\geq 24<36$ months. We compared the proportions of (1) fever development, (2) fever onset of $\geq 38.5^{\circ} \mathrm{C}$, and (3) the highest body temperature of $\geq 38.5^{\circ} \mathrm{C}$ among age groups. Differences among age groups were examined by calculating odds ratios. Significance was tested by Fisher's exact test. Significance level was set at $\mathrm{p}<0.05$.

A correlation between fever duration and age was examined using a regression equation after square root transformation of fever duration days, as a tool for stabilizing the variance. The strength of the correlation was estimated using Pearson's correlation coefficient. Significance level was set at $\alpha<0.05$.

\section{Concurrent clinical investigations}

Our collaborators collected blood samples at the acute and convalescent phase in order to investigate immunologic status. These data have been analyzed and reported in another publication (Jounai et al. 2017).

\section{Ethical issues}

This study was conducted following the principles of the Declaration of Helsinki. Each patient's caregiver provided written informed consent for study participation. In six of the participating hospitals (Yokosuka Kyosai Hospital, Yokohama Rosai Hospital, Yokohama City Minato Red Cross Hospital, Yokohama City University Medical Center, Yamato Municipal Hospital, and Yokohama Minami Kyosai Hospital) and Daiichi Sankyo CO., Ltd., the protocol was reviewed and approved by their institutional review boards. For three clinics (Sumita Kodomo Clinic, Maehara Pediatric Clinic, and Ohisama Kodomo Clinic), approval was by the institutional review board at Yokohama City University Medical Center.

\section{Results}

\section{Patients' characteristics and clinical symptoms}

A total of 179 children was enrolled into this study. Information on patient characteristics, fever onset and body temperature records were available on 171 of these 179 patients, who were then included in the analysis. There were 54 participants in the group of $<6$ months old, 41 in the group of $\geq 6<12$ months old, 58 in the group of $\geq 12$ $<24$ months old, and 18 in the group of $\geq 24<36$ months old.

Of the 171 participants, $10(6 \%)$ had an underlying disease; 8 of these $(5 \%)$ with respiratory disease ( 5 bronchial asthma, 2 asthmatic bronchitis, and one unspecified), one $(0.6 \%)$ with renal dysfunction and one with West syndrome.

Sex, median ages, and clinical symptoms in each age group are shown in Table 1. Cough and nasal discharge were the major acute symptoms, which remained about $20-30 \%$ during the convalescent phase in all age groups. During the acute phase, loss of appetite was present in about $10 \%$ of patients under 6 months old, but about $40-50 \%$ in patients of $>6$ months old. Other symptoms during the acute phase included wheezing or asthma in three subjects, diarrhea in two, and dehydration, vomiting, impaired consciousness and runny eyes in one. 
Table 1. Patients' characteristics and clinical symptoms.

\begin{tabular}{llllll}
\hline Age group & Total & $<6$ mo. & $\geq 6<12$ mo. & $\geq 12<24$ mo. $\geq 24<36$ mo. \\
& $(\mathrm{n}=171)$ & $(\mathrm{n}=54)$ & $(\mathrm{n}=41)$ & $(\mathrm{n}=58)$ & $(\mathrm{n}=18)$ \\
\hline \hline & $10(5,18)$ & $3.5(2,5)$ & $8(7,9)$ & $17(15,20)$ & $29(26,30)$ \\
Median month age (IQR) & $93(54)$ & $30(56)$ & $23(56)$ & $32(55)$ & $8(44)$ \\
\hline
\end{tabular}

Symptoms during acute phase. n (\%)

\begin{tabular}{|c|c|c|c|c|c|}
\hline Cough & 169 (99) & $53(98)$ & $41(100)$ & $57(98)$ & $18(100)$ \\
\hline Nasal discharge & $158(92)$ & $49(91)$ & $40(98)$ & $52(90)$ & $17(94)$ \\
\hline Loss of appetite & $56(33)$ & 7 (13) & $16(39)$ & $24(41)$ & $9(50)$ \\
\hline Others & $9(5)$ & $1(2)$ & $3(7)$ & $5(9)$ & $0(0)$ \\
\hline \multicolumn{6}{|c|}{ Symptoms during convalescent phase. n (\%) } \\
\hline Cough & $38(22)$ & $8(15)$ & $7(17)$ & $18(31)$ & $5(28)$ \\
\hline Nasal discharge & $56(33)$ & $11(20)$ & $13(32)$ & $23(40)$ & $9(50)$ \\
\hline Loss of appetite & $1(1)$ & $0(0)$ & $1(2)$ & $0(0)$ & $0(0)$ \\
\hline Others & $4(2)$ & $1(2)$ & $2(5)$ & $1(2)$ & $0(0)$ \\
\hline
\end{tabular}

\section{Fever responses}

Proportions of patients who experienced fever during the period of body temperature recording are shown in Fig. 1a for each age group. Fever occurred in about $70 \%$ in patients under 6 months old, which was significantly lower than in the group of $<12$ months and the group of $<24$ months. The odds ratios (ORs) and 95\% confidence intervals (CIs) for the $<6$ months versus the $<12$ months, the $<24$ months, and the $\geq 24$ months were 3.6 (95\% CI 1.111.7), 42.7 (95\% CI 2.48-733), and 6.5 (95\% CI 0.8-53.5), respectively. The proportion of patients who experienced fever gradually increased with advancing age up to the age of 24 months.

Proportions of high fever at onset $\left(\geq 38.5^{\circ} \mathrm{C}\right)$ among the 171 enrolled subjects are shown in Fig. 1b. High fever at onset was experienced by $<10 \%$ of patients under 6 months of age. This proportion was significantly lower than the other age groups. The ORs for the age group of $<6$ months versus the age group of $<12$ months, the age group of $<24$ months, and the age group of $>24$ months were 9.8 (95\% CI 3.0-32.2), 12.5 (95\% CI 4.1-39.1), and 8.0 (95\% CI 2.0-32.0), respectively. The proportion of patients with high fever at onset increased with advancing age up to 24 months of age.

Proportions of patients developing the highest body temperature of $\geq 38.5^{\circ} \mathrm{C}$ in each age group are shown in Fig. 1c. The proportion of patients with the highest fever of $\geq 38.5^{\circ} \mathrm{C}$ was about $25 \%$ in the $<6$ months age group, which is significantly lower than the $<12$ and the $<24$ months age groups. The ORs for age group of $<6$ months versus the age group of $<12$ months, $<24$ months, and $\geq 24$ months were 4.0 (95\% CI 1.7-9.6), 9.0 (95\% CI 3.8$21.1)$ and 2.3 (95\% CI $0.8-7.0)$, respectively. The proportion of the highest body temperature of $\geq 38.5^{\circ} \mathrm{C}$ increased 
(a) Fever development

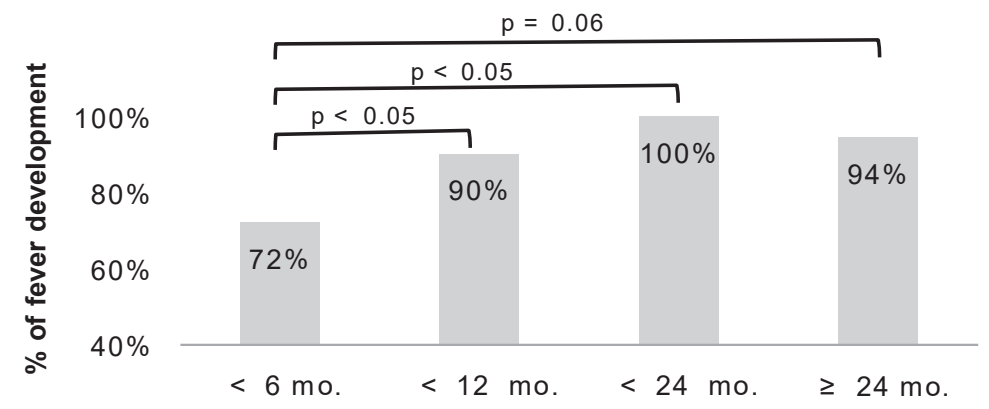

(b) Fever onset of $\geq 38.5^{\circ} \mathrm{C}$

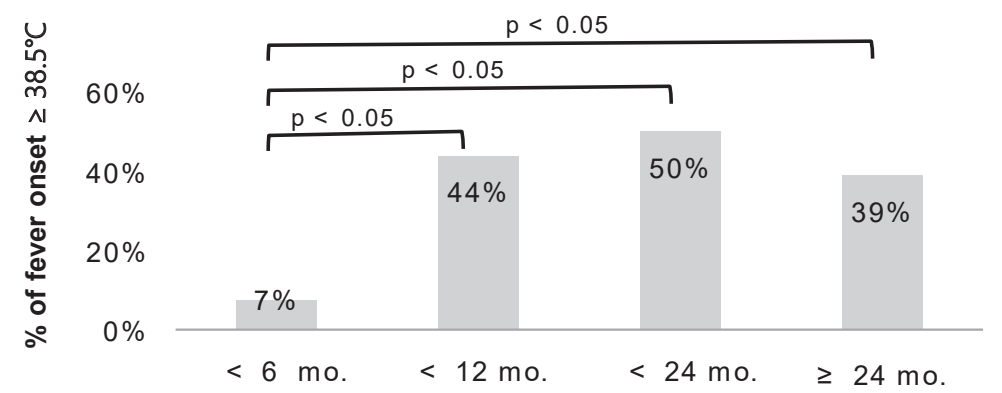

(c) The highest body te mperature of $\geq 38.5^{\circ} \mathrm{C}$

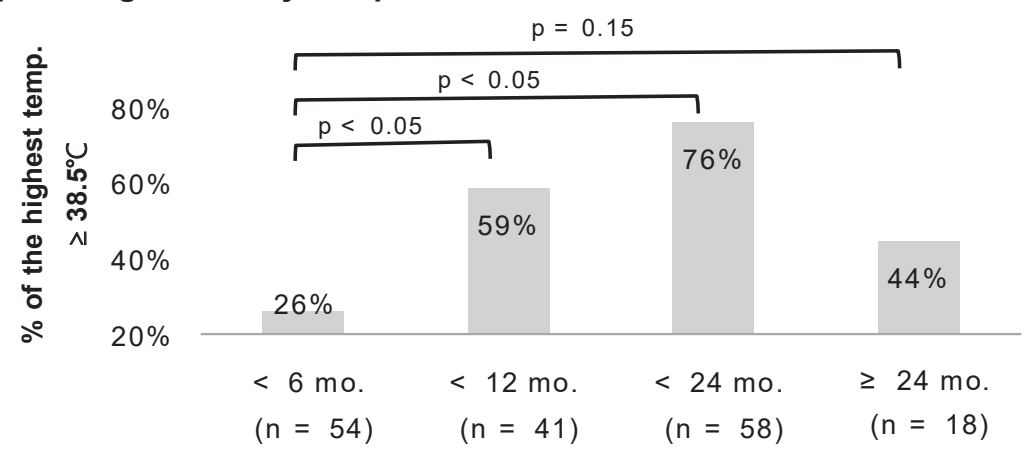

Age groups

Fig. 1. Fever responses.

Proportions of patients who developed fever, fever onset of $\geq 38.5^{\circ} \mathrm{C}$, and the highest temperature of $\geq 38.5^{\circ} \mathrm{C}$ in each age group are shown in (a), (b), and (c), respectively. P-values by Fisher's exact between the age-group $<6$ months old and other age groups are shown above the bars. Numbers of patients in each age group were 54 in the group of $<6$ months old, 41 in the group of $<12$ months old, 58 in the group of $<24$ months old, and 18 in the group of $\geq 24$ months old. Patients of $<6$ months old less frequently experienced fever (a), fever onset of $\geq 38.5^{\circ} \mathrm{C}$ (b), and the highest body temperature of $\geq 38.5^{\circ} \mathrm{C}$ (c), compared with other age groups, especially with those of $<12$ months old and those of $<$ 24 months old. Up to 24 months of age, proportions of patients who experienced fever, fever onset of $\geq 38.5^{\circ} \mathrm{C}$, and the highest body temperature of $\geq 38.5^{\circ} \mathrm{C}$ gradually increased with advancing age.

with advancing age up to 24 months. In patients of $\geq 24$ months old, even in those who developed fever, it often stayed within the range between $37.5^{\circ} \mathrm{C}$ and $38.4^{\circ} \mathrm{C}$.

There were 115 patients who did develop fever for more than one day. The median (ranges) of fever duration was 5 (range 1-14) days in the 115 patients. Broken down by age, median duration of fever days were 3 (range 1-7) days in the group of $<6$ months old, 5 (range 2-9) days in the group of $<12$ months old, 6 (range 2-14) days in the group of $<24$ months old, and 3.5 (range 1-7) days in the group of $\geq 24$ months old. The correlation between fever duration and age was examined in these patients (Fig. 2). Duration of fever was significantly longer with advancing age in patients under $<24$ months old. For those of $\geq 24$ months old, duration of fever did not tend to prolong further. 


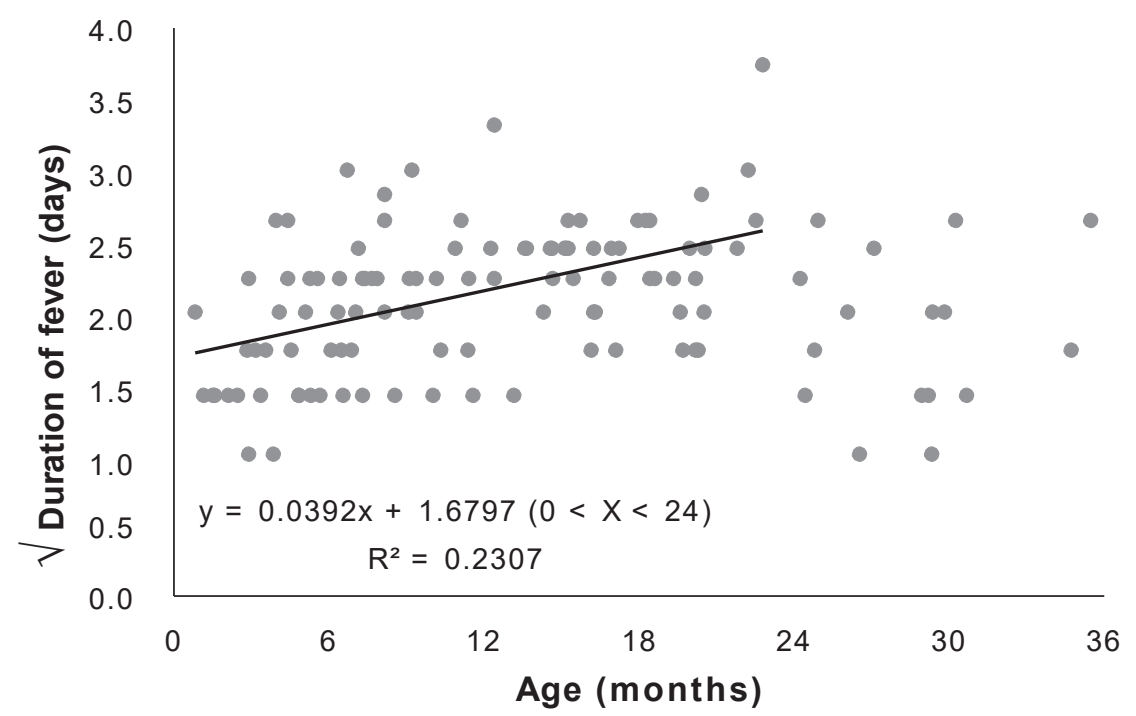

Fig. 2. Duration of fever.

Correlation between age and the square root of fever duration is shown. The 115 subjects, who had fever for more than one day, were included in this analysis. Numbers of subjects in each age group were 25 in the group of $<6$ months, 31 in the group of $<12$ months, 43 in the group of $<24$ months, and 16 in the group of $\geq 24$ months. Fever durations tended to prolong as age advanced up to 24 months old. A regression equation for subjects of $<24$ months resulted in estimates $( \pm \mathrm{SD})$ of slope and intercept as $0.039( \pm 0.007)$ and $1.68( \pm 0.09)$, respectively. Pearson's correlation coefficient in this regression equation was $0.48(\alpha<0.01)$, which meant significant correlation between age and fever duration among subjects of $<24$ months old. No correlation between age and fever duration was seen in patients of $\geq 24$ months old.

\section{Discussion}

In the present study, we monitored fever responses during RSV infection among children $<3$ years of age. Our study revealed that there were age-related fever responses, in which expression and durations of fever were enhanced with advancing age in children under $<24$ months of age.

In general, detections of viral invasion by innate immune cells lead to production of fever-generating factors (Mouihate et al. 2008; Evans et al. 2015). However, in early infancy, innate immunity is immature and poorly responsive due to residual in utero immune tolerance and lack of exposure to pathogens, infants at early months of age tend to suppress fever generation and maintenance (Arruvito et al. 2015; Goenka and Kollmann 2015). This concept was consistent with our findings, in which children of $<6$ months old tended to have reduced fever responses.

Attenuated fever responses impede the shift from innate immunity to acquired immunity. Hence, Th1 responses, one of acquired immune responses that play a major defense against viral infection, turn to be less effective (Lukens et al. 2010). Humoral immune responses, another acquired immunity where neutralizing antibodies are produced, are also less activated. A concurrent study conducted in the same subjects as this study showed consistent results; namely, the titer of neutralizing antibody produced in response to RSV infection in children under 6 months old was significantly low, and children's antibody responses against RSV are matured over months and years (Jounai et al. 2017). Due to such immature immunity in early infancy, virus elimination is protracted; as a result, prolonged disease duration may lead to severe consequences.

Children of $>24$ months old are considered to have already experienced their first RSV infection; also, their more matured immunity is expected to be more responsive and memorable than in early infancy. Such development in the children of $>24$ months old might be one of the reasons explaining the difference of fever responses, compared with other age groups.

The present study has some limitations. First, because the subjects were recruited at clinics or hospitals, they may not be generally representative of all children with RSV infection. Among children with mild symptoms, the older the child is, the less likely it is that a doctor will be consulted; on the other hand, younger children may be taken to doctors with symptoms of lesser severity. However, we believe that our findings at least indicate a tendency among those patients who did need medical consultation. Second, differences in treatment approaches were not studied, and the contribution of their efficacy for improving symptoms is unclear. That is, mixed infection with bacteria and combination with otitis media are often observed in RSV infection; thus, the use or non-use of antibiotics might also influence the duration of fever. Third, the form of disease manifestation, such as rhinosinusitis, bronchiolitis, or pneumonia, was not taken into consideration for each case in this study, which could have affected the documented fever course.

Based on the results of our study, it should be 
reminded that reduced fever responses in early infancy during RSV infection does not implicate less severity. Whether there is another early clinical sign indicating risks of potential severity among those young infants with muted fever responses is the next clinical question to be assessed. An approach we can take at present to reduce the RSV disease burden is to closely follow-up young children at high risk through early diagnosis, and to provide immediate treatment as required.

\section{Acknowledgments}

The authors thank Dr. Mayu Yamamoto, Dr. Satomi Higashi, Dr. Osamu Uchida, and Dr. Miho Sato of Yokosuka Kyosai Hospital, and Dr. Takeo Nakano of Nakano Pediatric Clinic for providing study patients. This study was supported by NexTEP grant (\#J13-09) from Japan Science and Technology Agency.

\section{Conflict of Interest}

This study was sponsored by Daiichi Sankyo Co., Ltd. Fumihiko Takeshita is an employee of Daiichi Sankyo Co., Ltd. Masaaki Mori received consulting fees from Daiichi Sankyo Co., Ltd. However, all authors declare no conflict of interest concerning the present study.

\section{References}

Amanatidou, V., Apostolakis, S. \& Spandidos, D.A. (2009) Genetic diversity of the host and severe respiratory syncytial virus-induced lower respiratory tract infection. Pediatr. Infect. Dis. J., 28, 135-140.

Arruvito, L., Raiden, S. \& Geffner, J. (2015) Host response to respiratory syncytial virus infection. Curr. Opin. Infect. Dis., 28, 259-266.

Crowe, J.E. Jr., (2016) RESPIRATORY SYNCYTIAL VIRUS. In Nelson Textbook of Pediatrics, 20th ed., edited by Kliegman, R.M., Stanton, B., Geme, J.S., Schor, N.F. Elsevier, Philadelphia, PA, pp. 1606-1609.

El Saleeby, C.M., Bush, A.J., Harrison, L.M., Aitken, J.A. \& Devincenzo, JP. (2011) Respiratory syncytial virus load, viral dynamics, and disease severity in previously healthy naturally infected children. J. Infect. Dis., 204, 996-1002.

Evans, S.S., Repasky, E.A. \& Fisher, D.T. (2015) Fever and the thermal regulation of immunity: the immune system feels the heat. Nat. Rev. Immunol., 15, 335-349.

Goenka, A. \& Kollmann, T.R. (2015) Development of immunity in early life. J. Infect., 71 Suppl 1, S112-120.

Hall, C.B., Weinberg, G.A., Blumkin, A.K., Edwards, K.M., Staat, M.A., Schlutz, A.F., Poehling, K.A., Szilagyi, P.G., Griffin, M.R., Williams, J.V., Zhu, Y., Grijalva, C.G., Prill, M.M. \& Iwane, M.K. (2013) Respiratory syncytial virus-associated hospitalizations among children less than 24 months of age. Pediatrics, 132, e341-348.

Hall, C.B., Weinbreg, G.A., Iwane, M.K., Blumkin, A.K., Edwards, K.M., Staat, M.A., Auinger, P., Griffin, M.R., Poehling, K.A., Erdman, D., Grijalva, C.G., Zhu, Y. \& Szilagyi, P. (2009) The burden of respiratory syncytial virus infection in young children. N. Engl. J. Med., 360, 588-598.

Jounai, N., Yoshioka, M., Tozuka, M., Inoue, K., Oka, T., Miyaji, K., Ishida, K., Kawai, N., Ikematsu, H., Kawakami, C., Shimizu, H., Mori, M., Ishii, K.J. \& Takeshita, F. (2017) Agespecific profiles of antibody responses against respiratory syncytial virus infection. EBioMedicine, 16, 124-135.

Legg, J.P., Hussain, I.R., Warner, J.A., Johnston, S.L. \& Warner, J.O. (2003) Type 1 and type 2 cytokine imbalance in acute respiratory syncytial virus bronchiolitis. Am. J. Respir. Crit. Care Med., 168, 633-639.

Lukens, M.V., van de Pol, A.C., Coenjaerts, F.E., Jansen, N.J., Kamp, V.M., Kimpen, J.L., Rossen, J.W., Ulfman, L.H., Tacke, C.E., Viveen, M.C., Koenderman, L., Wolfs, T.F. \& van Bleek, G.M. (2010) A systemic neutrophil response precedes robust $\mathrm{CD} 8^{+} \mathrm{T}$-cell activation during natural respiratory syncytial virus infection in infants. J. Virol., 84, 2374-2383.

Mella, C., Suarez-Arrabal, M.C., Lopez, S., Stephens, J., Fernandez, S., Hall, M.W., Ramilo, O. \& Mejias, A. (2013) Innate immune dysfunction is associated with enhanced disease severity in infants with severe respiratory syncytial virus bronchiolitis. J. Infect. Dis., 207, 564-573.

Mouihate, A., Harré, E.M., Martin, S. \& Pittman, Q.J. (2008) Suppression of the febrile response in late gestation: evidence, mechanisms and outcomes. J. Neuroendocrinol., 20, 508-514.

Welliver, R.C. (2000) Immunology of respiratory syncytial virus infection: eosinophils, cytokines, chemokines and asthma. Pediatr. Infect. Dis. J., 19, 780-783. 